\title{
Improved CT angiography protocols minimize radiation exposure
}

Published online: 18 June 2012

(C) Springer Healthcare 2012

MedWire News: Refinements to coronary computed tomography angiography (CCTA) practices can significantly reduce the radiation dose associated with imaging, demonstrates research from Massachusetts General Hospital in Boston, USA.

"The approximately 75\% dose reduction was achieved through a combination of factors, and at first glance our data might suggest that the reductions resulted from new CT purchases," say Leif-Christopher Engel and co-authors.

"However, our analyses reveal that although newer scanners contributed to dose reductions, a significant amount was due to increasing physician selection... of dose-protection methods.

"We speculate that these effects were facilitated by general advances in knowledge and by our site protocols, because during the entire study period, highly trained subspecialist physicians supervised each scan."

The median dose to the 1277 patients who underwent CCTA decreased by a significant $74.8 \%$ between 2005 and 2010. This represents a decrease from $13.1 \mathrm{mSv}$ in period 1, when a 64-slice multidetector CT was used without default protocols, to $3.3 \mathrm{mSv}$ in period 4 , where 128 -slice dual-source CT was used with body mass index and heart rhythm-based protocols.

The greatest dose reductions were achieved in period 4 by the adoption of axial-sequential acquisition, high-pitch helical acquisition, reduced tube voltage, and use of automatic exposure control, the team reports in the American Journal of Medicine.

"Our results suggest that simplified protocols can result in significantly decreased radiation burden and high diagnostic image quality," Engel et al conclude.

"This finding has implications for all practice settings from academic centers to community practices without the steady presence of a physician during the scan."

By Lynda Williams

\section{Reference}

Am J Med 2012; Advance online publication 